\title{
Bi-ventricular repair of Double Outlet Left ventricle - Experience and Review of Literature
}

\author{
Javid Raja ${ }^{1}$, Sabarinath Menon ${ }^{1}$, Sowmya Ramanan ${ }^{2}$, Sudip baruah ${ }^{1}$, Bhargava \\ Devarakonda $^{1}$, Arun Gopalakishnan ${ }^{1}$, and Baiju Dharan ${ }^{1}$ \\ ${ }^{1}$ Sree Chitra Tirunal Institute for Medical Sciences and Technology \\ ${ }^{2}$ Affiliation not available
}

May 20, 2020

\begin{abstract}
Double-outlet left ventricle (DOLV) is a rare congenital cardiac anomaly. The aorta and the main pulmonary arterial trunk arises predominantly from the left ventricle(LV) and is associated with a malaligned ventricular septal defect(VSD), various degrees of hypoplasia of the right ventricle, and presence or absence of pulmonary stenosis. Bi-ventricular repair is the preferred treatment option whenever possible. Various techniques for bi-ventricular repair have been described. The best option for DOLV correction is by translocating the pulmonary root to the right ventricle(RV)[1]. In this series, we report four patients who underwent biventricular repair of DOLV in our institute with excellent outcomes. All patient details were collected from the institute patient record system. Echocardiographic data were obtained from the records. Intraoperative charts were reviewed for further information on the surgical procedure and cardiopulmonary bypass. Postoperative data included survival, functional status and followup echocardiography. Of the four children, three underwent pulmonary root translocation and one child underwent Reparation al etage Ventriculaire(REV) procedure. There was no mortality and all children are in stable clinical condition in the recent follow-up and no re-operations or interventions were required following primary surgical correction. Thus DOLV is anatomically and surgically a challenging subset. Pulmonary root translocation in this anatomy is technically challenging but safe and superior option when compared to other alternative surgical procedures and it can be performed with excellent results, even in infants.
\end{abstract}

\section{Hosted file}

DOLV new.edited.pdf available at https://authorea.com/users/324378/articles/452589-biventricular-repair-of-double-outlet-left-ventricle-experience-and-review-of-literature 
Table 1 Patient characteristics and Intra-operative details

\begin{tabular}{|c|c|c|c|c|c|c|c|c|c|}
\hline $\begin{array}{l}\text { PT. } \\
\text { NO }\end{array}$ & AGE & SEX & $\begin{array}{l}\text { PREOP DIAGNO- } \\
\text { SIS }\end{array}$ & $\mid \begin{array}{c}\text { INTRAOP } \\
\text { MORPHOLOGY }\end{array}$ & VSD & $\begin{array}{l}\text { CORONARY } \\
\text { ANATOMY }\end{array}$ & PROCEDURE DONE & \begin{tabular}{|l} 
CPB \\
TIME \\
(MIN)
\end{tabular} & $\begin{array}{l}\text { CROSS- } \\
\text { CLAMP } \\
\text { TIME } \\
\text { (MIIN) }\end{array}$ \\
\hline 1 & $\begin{array}{c}1 \\
\text { MON }\end{array}$ & $\mathrm{F}$ & $\begin{array}{l}\text { dTGA WITH } \\
\text { 6MM MUSCU- } \\
\text { LAR VSD, } \\
\text { MILD PR, SE- } \\
\text { VERE PAH }\end{array}$ & \begin{tabular}{|c|} 
DOLV WITH \\
SIDE BY SIDE \\
GREAT AR- \\
TERIES, \\
AORTA TO \\
THE RIGHT \\
OF PA \\
\end{tabular} & SUBAORTIC & 1R2LCX & $\begin{array}{c}\text { VSD CLOSURE } \\
\text { WITH PULMO- } \\
\text { NARY ROOT } \\
\text { TRANSLOCATION }\end{array}$ & 383 & 230 \\
\hline 2 & $12 \mathrm{Y}$ & M & $\begin{array}{l}\text { DORV WITH } \\
\text { SEVERE PS, } \\
\text { GOOD PA } \\
\text { ANATOMY }\end{array}$ & \begin{tabular}{|c|} 
DOLV WITH \\
SIDE BY SIDE \\
GREAT AR- \\
TERIES, \\
AORTA TO \\
THE RIGHT \\
OF PA \\
\end{tabular} & SUBAORTIC & 1RLCX & $\begin{array}{l}\text { VSD CLOSURE } \\
\text { WITH PULMO- } \\
\text { NARY ROOT } \\
\text { TRANSLOCATION }\end{array}$ & 234 & 190 \\
\hline 3 & $3 \mathrm{Y}$ & M & $\begin{array}{l}\text { DORV WITH } \\
\text { SEVERE PS, } \\
\text { GOOD PA } \\
\text { ANATOMY, } \\
\text { LAD CROSS- } \\
\text { ING RVOT }\end{array}$ & \begin{tabular}{|c|} 
DOLV WITH \\
SIDE BY SIDE \\
GREAT AR- \\
TERIES, \\
AORTA TO \\
THE RIGHT \\
OF PA, PUL- \\
MONARY \\
VALVE BI- \\
CUSPID \& \\
STENOTIC
\end{tabular} & SUBAORTIC & $1 \mathrm{LCX} 2 \mathrm{R}$ & $\begin{array}{c}\text { VSD CLOSURE } \\
\text { WITH REV PROCE- } \\
\text { DURE WITH } \\
\text { MONOCUSP RE- } \\
\text { CONSTRUCTION } \\
\text { OF RVOT }\end{array}$ & 173 & 129 \\
\hline 4 & $\begin{array}{c}2 \\
\text { MON }\end{array}$ & $\mathrm{F}$ & $\begin{array}{l}\text { dTGA WITH } \\
\text { SIDE BY SIDE } \\
\text { GREAT AR- } \\
\text { TERIES, } \\
\text { LARGE } \\
\text { SUBAORTIC } \\
\text { VSD, MILD PR }\end{array}$ & \begin{tabular}{|c|} 
DOLV WITH \\
SIDE BY SIDE \\
GREAT AR- \\
TERIES, \\
AORTA TO \\
THE RIGHT \\
OF PA
\end{tabular} & SUBAORTIC & $\begin{array}{c}\text { 1LR2CX } \\
\text { WITH } \\
\text { DOUBLE } \\
\text { LOOPING } \\
\text { OF CORO- } \\
\text { NARY } \\
\text { AROUND } \\
\text { PA }\end{array}$ & $\begin{array}{l}\text { VSD CLOSURE } \\
\text { WITH PULMO- } \\
\text { NARY ROOT } \\
\text { TRANSLOCATION }\end{array}$ & 214 & 152 \\
\hline
\end{tabular}


Table 2 Latest follow-up Echocardiographic Findings

\begin{tabular}{|c|c|c|c|c|c|c|c|c|}
\hline Patient no. & $\begin{array}{c}\text { RVOT GRAD } \\
\text { PEAK } \\
\text { (mmHg) }\end{array}$ & PR & TAPSE & RVSP & LVOT GRAD & LVEF & $\begin{array}{c}\text { ROSS MOD- } \\
\text { IFED } \\
\text { SCORE }\end{array}$ & $\begin{array}{c}\text { FOLLOWUP } \\
\text { (MONTHS) }\end{array}$ \\
\hline 1 & 12 & 2 & 19 & 25 & 16 & 68 & 1 & 17 \\
\hline 2 & 10 & 1 & 18 & 35 & 16 & 70 & 1 & 15 \\
\hline 3 & 22 & 2 & 18 & 28 & 10 & 66 & 2 & 12 \\
\hline 4 & 16 & NIL & 20 & 45 & 15 & 68 & 1 & 10 \\
\hline
\end{tabular}

PR - PULMONARY INCOMPETENCE, TAPSE - TRICUSPID ANNULAR PLANE SYSTOLIC

EXCURSION, RVSP - RIGHT VENTRICLE SYSTOLIC PRESSURE, LVOT GRAD - LEFT

VENTRICLE OUTFLOW TRACT GRADIENT, LVEF - LEFT VENTRICLE EJECTION FRACTION 
Table 3 Literature review of Pulmonary root translocation

\begin{tabular}{|l|c|c|c|l|c|l|l|}
$\begin{array}{l}\text { Au- } \\
\text { thor/year }\end{array}$ & $\begin{array}{c}\text { NUMBER } \\
\text { OF PA- } \\
\text { TIENTS }\end{array}$ & VSD & $\begin{array}{c}\text { PULMONARY } \\
\text { OUTFLOW OB- } \\
\text { STRUCTION }\end{array}$ & $\begin{array}{l}\text { GREAT ARTER- } \\
\text { IES RELATION- } \\
\text { SHIP }\end{array}$ & $\begin{array}{l}\text { CORONARY } \\
\text { ANOMALIES }\end{array}$ & $\begin{array}{l}\text { PROCEDURE } \\
\text { DONE }\end{array}$ & OUTCOME \\
\hline $\begin{array}{l}\text { Chiavarelli } \\
\text { et al. 1992 }\end{array}$ & $\mathbf{1}$ & Subaortic & YES & S, D, D & YES & $\begin{array}{l}\text { PULMONARY } \\
\text { ROOT TRANSLO- } \\
\text { CATION }\end{array}$ & UNEVENTFUL \\
\hline $\begin{array}{l}\text { DeLeon et } \\
\text { al. 1995 }\end{array}$ & $\mathbf{2}$ & Subaortic & YES & S, D, D & YES & $\begin{array}{l}\text { PULMONARY } \\
\text { ROOT TRANSLO- } \\
\text { CATION }\end{array}$ & UNEVENTFUL \\
\hline $\begin{array}{l}\text { McElhinney } \\
\text { et al. 1997 }\end{array}$ & $\mathbf{3}$ & Subaortic & YES & S, D, D & NO & $\begin{array}{l}\text { PULMONARY } \\
\text { ROOT TRANSLO- } \\
\text { CATION }\end{array}$ & UNEVENTFUL \\
$\begin{array}{l}\text { Ootaki et al. } \\
\text { 2001 }\end{array}$ & $\mathbf{2}$ & Subaortic & YES & S, D, D & NO & $\begin{array}{l}\text { PULMONARY } \\
\text { ROOT TRANSLO- } \\
\text { CATION }\end{array}$ & UNEVENTFUL \\
\hline
\end{tabular}






posiat Xute

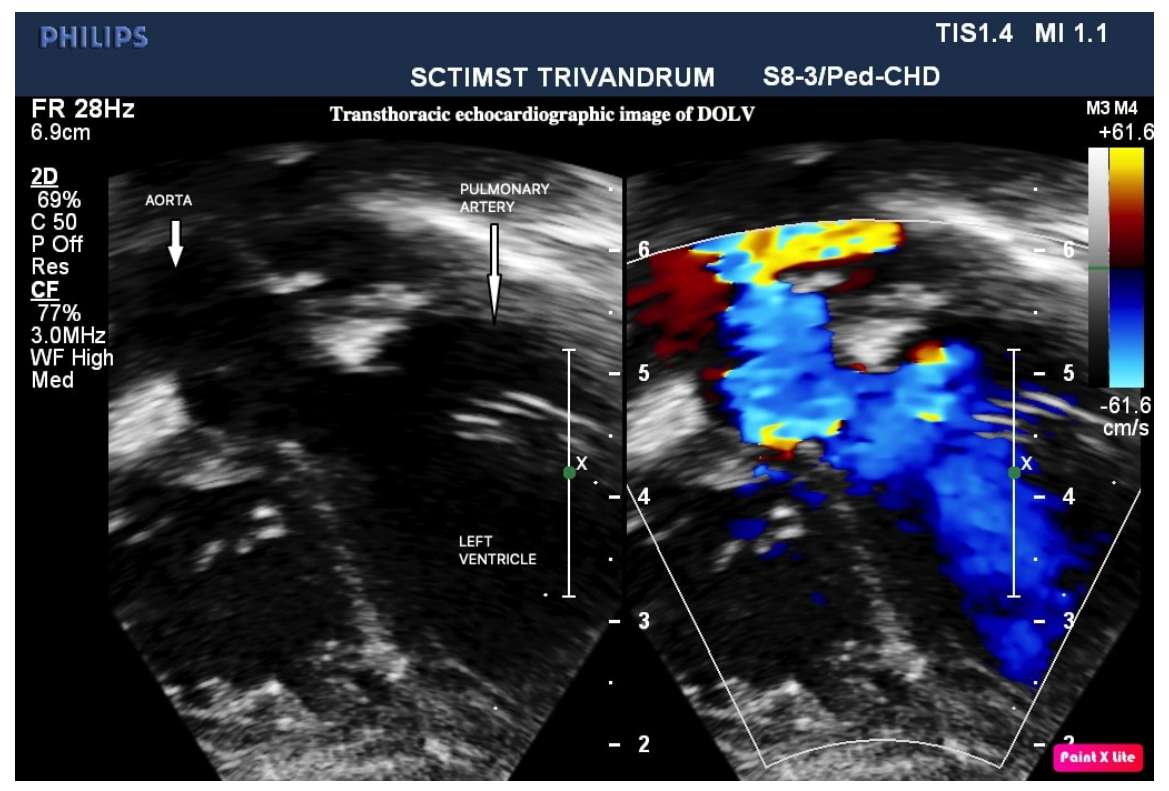

